
\title{
R Reserchch Suare \\ HDAC Inhibitor 4SC-202 Increases Sensitivity to Epirubicin in Liver Cancer Cells by Inhibition of ATM
}

\section{Zhixiang Cheng}

Wuhan University Zhongnan Hospital

Quan Sun

Wuhan University Zhongnan Hospital

\section{Ping Jiang}

Wuhan University Zhongnan Hospital

Bo Liao ( $D$ wudaliaobo@126.com)

Wuhan University Zhongnan Hospital

\section{Research}

Keywords: 4SC-202, Epirubicin, Liver cancer, HDAC, ATM, TPD52

Posted Date: May 6th, 2021

DOI: https://doi.org/10.21203/rs.3.rs-490086/v1

License: (c) (1) This work is licensed under a Creative Commons Attribution 4.0 International License. Read Full License 


\section{Abstract}

Background: Histone deacetylase (HDAC) inhibitors can enhance the anticancer effect of epirubicin in liver cancer. However, the molecular mechanism remains unknown. In our study, we aimed to examine the effect of the combination of HDAC inhibitor 4SC-202 and epirubicin and the underlying mechanism in liver cancer cells.

Methods: Cell viability assay, flow cytometry, western blotting, colony formation assay, animal experiment, immunohistochemistry, PCR, and chromatin immunoprecipitation were conducted.

Results: We found that 4SC-202 and epirubicin inhibited the growth and induced cell cycle arrest and apoptosis in of HepG2 and Huh7 cells. The combination treatment also reduced tumorigenicity of liver cancer cells in vitro and in vivo. Moreover, 4SC-202 promoted tumor protein D52 (TPD52) transcription and induced TPD52 expression by inhibiting HDAC1/2. TPD52 downregulated ataxia telangiectasia mutated (ATM) expression caused by epirubicin, and liver cancer cells were more sensitive to epirubicin after ATM inhibition.

Conclusions: Our results show that 4SC-202 enhances chemosensitivity to epirubicin in liver cancer cells via inhibiting ATM and suggest that the combination may serve as a novel strategy for treatment of liver cancer.

\section{Introduction}

Liver cancer is the third leading cause of death and a major public health problem in China [1]. In 2007, sorafenib was approved by the FDA for the treatment of liver cancer and is currently the first-line drug for liver cancer [2]. However, sorafenib is often associated with drug resistance and serious adverse side effects, limiting its clinical application [2]. Therefore, the development of new effective strategies for liver cancer treatment is required.

Epirubicin (Fig. 1a) is a type of anthracycline and shows cytotoxic effects on a variety of solid tumors [3, 4]. Epirubicin induces DNA double-strand breaks (DSBs), causing toxic DNA damage and cell death [3]. Previous studies reported that epirubicin shows less cardiotoxicity compared with doxorubicin, another anthracycline $[3,5]$. However, the clearance of DSBs and DNA damage repair leads to chemoresistance to epirubicin, which hampers the clinical use of this agent $[3,5]$.

HDAC inhibitors are a series of chemicals targeting HDACs that show anticancer effects in many types of cancer [6-8]. Four HDAC inhibitors have been approved by the FDA for cancer treatment, and several clinical trials of HDAC inhibitors in liver cancer are ongoing $[9,10]$. 4SC-202 (domatinostat) (Fig. 1b) is a novel selective class I HDAC inhibitor (HDACi) that mainly targets HDAC1/2/3 [11]. This compound has been reported to exert antineoplastic effects on various types of cancer in vitro and in vivo [12-17]. A previous study showed that 4SC-202 exhibits antitumor activity in liver cancer by activating the ASK1dependent mitochondrial apoptosis pathway [18]. A phase I study demonstrated that patients with 
advanced hematological malignancies benefited from 4SC-202 administration [19]. Some studies have shown benefits of combination anticancer drug treatments including HDACi. We previously reported that another $\mathrm{HDACi}$, vorinostat, enhanced chemosensitivity to anticancer agents such as 5-fluorouracil and oxaliplatin in liver cancer $[7,8]$. The HDACi valproic acid also augmented the cytotoxic effect of epirubicin on liver cancer HepG2 cells [20].

In this study, we investigated the anticancer effect of the combination administration of 4SC-202 and epirubicin on liver cancer cells. The underlying mechanism of the combination treatment was also explored.

\section{Materials And Methods}

\section{Cell lines, cell culture, and reagents}

The HepG2 hepatoma cell line and Huh7 hepatocellular carcinoma cell line were purchased from China Center for Type Culture Collection (CCTCC, Wuhan, China). Both cell lines were cultured in high-glucose DMEM (Hyclone, Logan, UT, USA) containing 10\% FBS (Gibco, Grand Island, NY, USA) at $37^{\circ} \mathrm{C}$ and $5 \%$ $\mathrm{CO}_{2}$. Epirubicin and 4SC-202 were purchased from Selleck Chemicals (Houston, TX, USA). Crystal violet and DMSO were purchased from Sigma-Aldrich (St. Louis, MO, USA). The CCK-8 kit was purchased from Dojindo (Kumamoto, Japan).

\section{Cytotoxicity assay, combination index $(\mathrm{Cl})$ analysis, and dose-reduction index (DRI) analysis}

Cytotoxicity assay was performed as described previously [7, 8]. Briefly, HepG2 (1,000 cells/well) and Huh7 (1,500 cells/well) cells were seeded and treated with increasing concentrations of 4SC-202 and epirubicin. IC50 values of these two drugs were calculated using SPSS software. Cl and DRI were analyzed as described previously to evaluate the synergistic effect of the two drugs $[7,8]$.

\section{Flow cytometry}

Cell cycle and apoptosis analysis was carried out as described previously [7, 8]. HepG2 and Huh7 cells were treated with 4SC-202 and epirubicin for $48 \mathrm{~h}$ and analyzed using a FACSAria Cell Cytometer (BD Biosciences, San Jose, CA, USA). Cell cycle distribution and apoptotic rate were analyzed using CellQuest software (BD Biosciences).

\section{Western blotting}

Western blotting was performed as described previously [7, 8]. Primary antibodies against acetylated histone H3 (AcH3), $\beta$-actin, and TPD 52 were purchased from Santa Cruz Biotechnology (Santa Cruz, CA, USA). Antibodies against PARP, ATM, PATM, HDAC1, HDAC2, and HDAC3 were purchased from Cell Signaling Technology (CST, Beverly, MA, USA). All secondary antibodies were purchased from Jackson Immuno Research Laboratories (West Grove, PA, USA). 


\section{Colony formation assay}

In vitro tumorigenicity assay was carried out as described previously [7, 8]. HepG2 (500 cells/well) and Huh7 (500 cells/well) cells were seeded in 6-well plates and treated with 4SC-202 and epirubicin. The numbers of colonies were analyzed using an Alpha Innotech Imaging system (Alphatron Asia Pte Ltd, Singapore).

\section{Animal studies}

Animal studies were performed as previously described [8]. All procedures in animal studies were approved by the Committee on the Ethics of Animal Experiments of Zhongnan Hospital, Wuhan University. Animal Experiments were carried out in the Animal Research Center of Wuhan University. HepG2 cells were subcutaneously injected into mice. At day 9 after injection, mice were treated with 4SC202, epirubicin or control and divided into 4 groups $(n=6) .4$ SC-202 $(100 \mathrm{mg} / \mathrm{kg})$ was dissolved in DMSO and dosed per os (p.o.) daily. Epirubicin ( $3 \mathrm{mg} / \mathrm{kg}$ ) was dissolved in DMSO and injected intraperitoneally (i.p.) every third day. On day 27 , all the animals were sacrificed by cervical dislocation. Tumors were harvested for growth and apoptosis analysis. Biochemical functions of liver and kidney including aspartate aminotransferase (AST), alanine aminotransferase (ALT), creatinine (Cr), and blood urea nitrogen (BUN) in serum were measured by the Reflotron Plus system (Roche, IN, USA). Organs such as lung, spleen, liver, kidney, and heart were sectioned for analyses.

\section{Immunohistochemistry analysis}

Immunohistochemistry was performed as previously described [7, 8]. Ki-67 antibody was purchased from Dako (Golstrup, Denmark) and cleaved caspase-3 was from CST.

\section{Quantitative PCR analysis}

PCR was performed as described previously [8]. The sequences of primers for ATM, TPD52, HDAC1, HDAC2, HDAC3, and ACTB genes are listed in Table 1. ACTB was used as an internal control for normalization. The relative change folds were analyzed using the $2^{-\Delta \Delta C q}$ method [21]. 
Table 1

Primer sequences for qPCR

\begin{tabular}{|ll|}
\hline Gene & Primer sequences \\
\hline ATM & F: 5'-GTGCCTAAACAAAGCTCTCAG-3' \\
\hline TPD52 & R: 5' 5'-CCTCAACACTTCTGACCATCT-3' $^{\prime}$-GAGAAGGAGAAGATGTTGC-3' \\
& R: 5'-GCCGAATTCAAGACTTCTCC-3' \\
\hline HDAC1 & F: 5'-GCTCCACATCAGTCCTTCC-3' \\
& R: 5'-GGTCGTCTTCGTCCTCATC-3' \\
HDAC2 & F: 5'-AGGCAAATACTATGCTGTC-3' \\
& R: 5'-TGAAACAACCCAGTCTATC-3' \\
\hline HDAC3 & F: 5'-CGGGATGGCATTGATGAC-3' \\
\hline & R: 5'- GGGCAACATTTCGGACAG-3' \\
\hline ACTB & F: 5'-CAAGGCCAACCGCGAGAAGAT-3' \\
\hline & R: 5'-CCAGAGGCGTACAGGGATAGCAC-3' \\
\hline
\end{tabular}

\section{RNA interference}

Knockdown of ATM, TPD52, HDAC1, HDAC2, and HDAC3 was carried out using siRNA. HDAC1, HDAC2, and HDAC3 siRNAs were purchased from Santa Cruz Biotechnology. The sequence for siATM 1 was $5^{\prime}-$ GCAAAGCCCUAGUAACAUA-3' and the sequence for siATM 2 was 5'-GGGCAUUACGGGUGUUGAA-3'. The sequence for siTPD52 1 was 5'- GCGGAAACUUGGAAUCAAU-3' and the sequence for siTPD52 2 was 5'GGAGAAGUCUUGAAUUCGG-3'. The siRNAs were synthesized by Ribobio (Guangzhou, China).

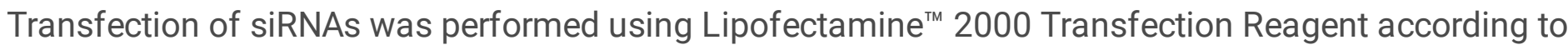
the manufacturer's instructions.

\section{Chromatin immunoprecipitation (ChIP) assay}

Quantitative ChIP assay was performed as described previously [22]. HepG2 cells were immunoprecipitated with anti-AcH3, anti-HDAC1, anti-HDAC2, or IgG. The primers for region A of the TPD52 promoter were 5'-CACCAGATTCAGGAGCCCAC-3' (forward) and 5'- GTCCCATCGATCACCCAAGG-3' (reverse). The primers for region B of the TPD52 promoter were 5'- AAGTGCTTGGATGACTGGCA-3' (forward) and 5'-GCGCAACCATCACCGTAATC-3' (reverse). The primers for region C of the TPD52 promoter were 5'-CCATGCTTAGTGGCAAGAGG-3' (forward) and 5'-GTTAGTTGCATCGTCAGCCT-3' (reverse).

\section{Statistical analysis}


SPSS 13.0 software (SPSS Inc., Chicago, IL, USA) was used to perform statistical analyses. All experiments were repeated at least three times. The final data were expressed as the mean \pm SD. Comparisons among the different groups were analyzed using one-way ANOVA, and $\mathrm{P}<0.05$ was considered as statistically significant.

\section{Results}

\section{SC-202 elevated the level of acetylated histone H3 in liver cancer cells}

We first examined the impact of the HDACi 4SC-202 on AcH3 level in two liver cancer cell lines. Western blotting result showed that 4SC-202 administration for $48 \mathrm{~h}$ enhanced the levels of AcH3 in HepG2 and Huh7 cell lines in a dose-dependent manner (Fig. 1c).

\section{SC-202 and epirubicin inhibited the proliferation of liver cancer cells}

We then explored the cytotoxic effect of 4SC-202 and epirubicin on the two liver cancer cell lines. HepG2 and Huh7 cells were treated with increasing concentrations of 4SC-202 or epirubicin. CCK-8 assays showed that 4SC-202 and epirubicin each exerted time-dependent and dose-dependent inhibitory effects on HepG2 and Huh7 cell proliferation (Fig. 1d and e). We also examined 4SC-202 in combination with epirubicin and found that all $\mathrm{Cl}$ values were lower than 1 (Fig. 1f) and all DRI values were higher than 1 (Fig. 1g), indicating a synergistic effect of the combination of 4SC-202 and epirubicin.

\section{SC-202 and epirubicin led to cell cycle arrest and apoptosis in liver cancer cells}

Epirubicin treatment resulted in S phase arrest in both HepG2 and Huh7 cells (Fig. 2a). Co-treatment with 4SC-202 reduced the epirubicin-mediated S phase arrest, promoting S phase slippage of epirubicintreated cells and inducing transition into G2/M phase (Fig. 2a). Both 4SC-202 and epirubicin caused apoptosis in HepG2 and Huh7 cells (Fig. 2b). Co-treatment with the two drugs significantly increased the apoptotic level compared with either 4SC-202 or epirubicin alone. Western blotting demonstrated that the expression of the apoptotic factor cleaved-PARP was elevated after 4SC-202 and/or epirubicin (Fig. 2c).

\section{SC-202 and epirubicin attenuated the tumorigenicity of liver cancer cells in vitro}

We next performed colony formation assay to evaluate the effect of 4SC-202 and epirubicin on the tumorigenicity of liver cancer cells. The results showed that 4SC-202 and epirubicin decreased both the size and number of colonies in HepG2 and Huh7 cells (Fig. 2d). Combined treatment of the two drugs significantly inhibited colony formation compared with treatment of 4SC-202 or epirubicin alone.

\section{SC-202 increased the antitumor effect of epirubicin in vivo}


We next evaluated the influence of 4SC-202 and epirubicin on the growth of liver cancer cells in an animal model. On day 27, mice were sacrificed. Treatment with 4SC-202 or epirubicin caused a slight decrease in tumor growth, while the combination led to a significantly increased reduction in tumor size and weight compared with the single treatments alone (Fig. 3a-c). Immunohistochemistry of Ki-67 and cleaved caspase-3 was used to analyze proliferation and apoptosis in tumor sections. Treatment with either 4SC202 or epirubicin caused a slight decrease in the percentage of Ki-67-positive cells and a slight increase in cleaved caspase-3-positive cells (Fig. 3d). Notably, the combination treatment led to a significant reduction of Ki-67-positive cells and a significant accumulation of cleaved caspase-3-positive cells compared with the single treatment groups. No differences in body weight of animals were observed among groups, indicating minimal drug toxicity or 4SC-202 and/or epirubicin treatment (Fig. 3e). Moreover, biochemical functions of liver and kidney were not affected by administration of the two drugs (Fig. 3f). No obvious histological changes were observed in the organs such as lung, spleen, liver, kidney, and heart (Fig. 3g). Therefore, the administration doses of 4SC-202 and epirubicin used in this study were safe and effective.

\section{SC-202 downregulated ATM expression caused by epirubicin in liver cancer cells}

Epirubicin treatment of in HepG2 induced elevation of both ATM mRNA and protein levels (Fig. 4a and b). In contrast, ATM mRNA and protein expressions were downregulated following 4SC-202 treatment (Fig. 4c and d). Co-treatment of both drugs showed that 4SC-202 reversed the effect on ATM upregulation caused by epirubicin (Fig. 4e and f).

To examine the role of ATM in epirubicin-mediated effects on liver cells, we inhibited ATM expression using siRNA and verified the knock-down by qPCR and western blotting (Fig. $4 \mathrm{~g}$ and $\mathrm{h}$ ). We found that after ATM inhibition, HepG2 cells were much more sensitive to epirubicin, indicating the role of ATM in the chemosensitivity of liver cancer cells to epirubicin (Fig. 4i).

\section{TPD52 was repressed by HDAC1/2 and downregulated ATM}

We next evaluated TPD52 expression after 4SC-202 and epirubicin treatment. The mRNA and protein expressions of TPD52 were upregulated by 4SC-202 but not influenced by epirubicin (Fig. 5a and b). We confirmed effective don regulation of TPD52 expression by siRNA (Fig. 5c). While ATM mRNA levels were not affected upon TPD52 knockdown, ATM protein level was upregulated in cells transfected with TPD52 siRNA (Fig. 5d and e), which was consistent with previous research in breast cancer cells [23].

Given that 4SC-202 mainly targets HDAC1/2/3, we next evaluated the impact of HDACs on TPD52 and ATM using HDAC1/2/3 siRNA. We used siHDAC1/2, siHDAC1/3, siHDAC2/3, and siHDAC1/2/3 to explore the differences among the above groups. We validated the knockdown effect of HDAC siRNAs (Fig. 5f-h). TPD52 mRNA was increased and ATM mRNA was decreased followed by HDAC1/2 inhibition (Fig. $5 \mathrm{i}$ and j). Western blot results of TPD52 and ATM levels were consistent with these results (Fig. 5k). 


\section{AcH3 and HDAC1/2 competitively bound to TPD52 promoter}

We next performed ChIP assay to evaluate whether $\mathrm{AcH} 3$ or $\mathrm{HDAC} 1 / 2$ directly bound to the promoter of the TPD52 gene. First, we designed three pairs of primers for the TPD52 promoter region (Fig. 6a and b). We observed strong binding of AcH3 to the TPD52 promoter region, and binding was significantly enriched with 4SC-202 treatment (Fig. 6c). We also detected binding of HDAC1/2 to the TPD52 promoter region, but binding of both HDACs was attenuated by 4SC-202 treatment (Fig. $6 \mathrm{~d}$ and e). These results suggest that 4SC-202 upregulates TPD52 expression by inhibiting HDAC1/2 and increasing AcH3 at the TPD52 promoter region.

\section{Discussion}

In this study, we investigated the effects of the combination treatment of the HDACi 4SC-202 with epirubicin on liver cancer cells. We found that 4SC-202 augmented AcH3 levels in liver cancer cells. The combination treatment of 4SC-202 and epirubicin synergistically inhibited the proliferation of liver cancer cells. Epirubicin caused S phase arrest and 4SC-202 partially reversed this effect; 4SC-202 mediated S phase slippage of cells after epirubicin treatment and led to transition into G2/M phase. We also found that 4SC-202 and epirubicin synergistically triggered apoptosis and showed that 4SC-202 and epirubicin synergistically attenuated the tumorigenicity of liver cancer cells in vitro and in vivo. Together these results indicate 4 SC-202 as an efficacious chemosensitizer of epirubicin in liver cancer cells.

As a DNA-damaging reagent, epirubicin causes DSBs, and the DNA damage then activates the ATM pathway. ATM plays a key role in regulation of the DNA repair. ATM phosphorylates and then activates BRCA1, BRCA2, FANCD2, BLM, Nbs1, Chk1, H2AX, WRN, p53, RPA, SMC1, and c-Abl to induce homologous recombination [24]. Consequently, ATM promotes chemoresistance to epirubicin, and our study showed that liver cancer cells were much more sensitive to epirubicin after ATM inhibition.

Previous studies demonstrated the synergistic effects of HDACi and epirubicin on various types of cancers such as breast cancer, gastric cancer, colorectal cancer, melanoma, lung cancer, and osteosarcoma in vitro and in vivo [25-28]. In a clinical trial, the HDACi valproic acid followed by epirubicin has shown promise as an effective treatment for advanced solid tumors [29]. Some studies showed that HDACi can function as a negative regulator of ATM pathway. The HDACi vorinostat decreases ATM expression via inhibition of HDAC1/2 in breast cancer [28]. In colorectal cancer, the HDACi MS-275 upregulates PR130 by inhibiting HDAC1/2 and PR130 hampers ATM pathway expression caused by hydroxyurea [30]. In B-cell-lymphoid neoplastic cells, valproic acid also reduces ATM level and increases ROS-dependent cell death when combined with fludarabine [31].

TPD52 is an inhibitor of ATM pathway [23]. A direct interaction between ATM and TPD52 protein was confirmed by co-immunoprecipitation and GST pull-down assays, and overexpression of TPD52 led to reduced ATM protein levels [23]. Previous studies reported an involvement of TPD52 in the tumorigenesis 
of liver cancer and TPD52 has bee considered as a tumor suppressor in liver cancer [27]. In our study, we hypothesized that 4SC-202 may upregulate TPD52 expression to inhibit ATM expression. We found that 4SC-202 decreased ATM expression upregulated by epirubicin and increased TPD52 expression.

Consistent with previous studies, we also found that TPD52 downregulated the protein level of ATM but not mRNA expression. Through knockdown experiments, we found that HDAC1/2 inhibited the mRNA and protein levels of TPD52 but induced those of ATM, suggesting that HDAC1/2 influenced the mRNA level of ATM independent of TPD52. The influence of HDAC1/2 on ATM transcription needs to be further explored. ChIP assay further demonstrated that $\mathrm{AcH} 3$ and $\mathrm{HDAC} 1 / 2$ competitively bound to the TPD52 promoter to influence its transcription.

In summary, our study showed that 4SC-202 enhanced the chemosensitivity of liver cancer cells to epirubicin (Fig. 7). 4SC-202 promotes TPD52 transcription and expression via inhibition of HDAC1/2. TPD52 attenuates ATM expression caused by epirubicin. These findings suggested that 4SC-202 may serve as a chemosensitizer to epirubicin for patients with liver cancer.

\section{Abbreviations}

HDAC: histone deacetylase; TPD52: tumor protein D52; ATM: ataxia telangiectasia mutated; DSBs: DNA double-strand breaks; HDACi: HDAC inhibitor; Cl: combination index; DRI: dose-reduction index; IC50: half maximal inhibitory concentration; AcH3: acetylated histone H3; AST: aspartate aminotransferase; ALT: alanine aminotransferase; Cr: creatinine; BUN: blood urea nitrogen; ChIP: chromatin immunoprecipitation

\section{Declarations}

\section{Acknowledgements}

Not applicable.

\section{Funding}

This study was supported by the Natural Science Foundation of Hubei Province of China (Grant No. 2019CFB707 \& 2020CFB708) and the Natural Science Foundation of China (Grant No. 81903001).

\section{Availability of data and materials}

The datasets used and/or analysed during the current study are available from the corresponding author on reasonable request.

\section{Ethics approval and consent to participate}

All procedures in animal experiments were approved by the Committee on the Ethics of Animal Experiments of Zhongnan Hospital, Wuhan University. 


\section{Competing interests}

The authors declare that they have no competing interests.

\section{Consent for publication}

Not applicable.

\section{Authors' contributions}

LB conceived of the presented idea. CZ, JP, and SQ developed the theory and performed the experiments. $\mathrm{CZ}$ and JP were responsible for data analysis and interpretation. LB supervised the findings of this work. All authors discussed the results and contributed to the final manuscript.

\section{References}

1. Chen W, Zheng R, Baade PD, Zhang S, Zeng H, Bray F, et al. Cancer statistics in China, 2015. CA Cancer J Clin. 2016;66:115-32.

2. Zhu YJ, Zheng B, Wang HY, Chen L. New knowledge of the mechanisms of sorafenib resistance in liver cancer. Acta Pharmacol Sin. 2017;38:614-22.

3. Cortes-Funes $\mathrm{H}$, Coronado $\mathrm{C}$. Role of anthracyclines in the era of targeted therapy. Cardiovasc Toxicol. 2007;7:56-60.

4. Pohl J, Zuna I, Stremmel W, Rudi J. Systemic chemotherapy with epirubicin for treatment of advanced or multifocal hepatocellular carcinoma. Chemotherapy. 2001;47:359-65.

5. Zhang X, Zhang Z, Zhang Q, Sun P, Xiang R, Ren G, et al. ZEB1 confers chemotherapeutic resistance to breast cancer by activating ATM. Cell Death Dis. 2018;9:57.

6. Eckschlager T, Plch J, Stiborova M, Hrabeta J. Histone Deacetylase Inhibitors as Anticancer Drugs. Int J Mol Sci. 2017;18.

7. Liao B, Liang H, Chen J, Liu Q, Zhang B, Chen X. Suberoylanilide hydroxamic acid enhances chemosensitivity to 5 -fluorouracil in hepatocellular carcinoma via inhibition of thymidylate synthase. Tumour Biol. 2015;36:9347-56.

8. Liao B, Zhang Y, Sun Q, Jiang P. Vorinostat enhances the anticancer effect of oxaliplatin on hepatocellular carcinoma cells. Cancer Med. 2018;7:196-207.

9. Tsilimigras DI, Ntanasis-Stathopoulos I, Moris D, Spartalis E, Pawlik TM. Histone deacetylase inhibitors in hepatocellular carcinoma: A therapeutic perspective. Surg Oncol. 2018;27:611-8.

10. Liu KY, Wang LT, Hsu SH. Modification of Epigenetic Histone Acetylation in Hepatocellular Carcinoma. Cancers (Basel). 2018;10.

11. Maes T, Carceller E, Salas J, Ortega A, Buesa C. Advances in the development of histone lysine demethylase inhibitors. Curr Opin Pharmacol. 2015;23:52-60. 
12. Pinkerneil M, Hoffmann MJ, Kohlhof H, Schulz WA, Niegisch G. Evaluation of the Therapeutic Potential of the Novel Isotype Specific HDAC Inhibitor 4SC-202 in Urothelial Carcinoma Cell Lines. Target Oncol. 2016;11:783-98.

13. Zhijun H, Shusheng W, Han M, Jianping L, Li-Sen Q, Dechun L. Pre-clinical characterization of 4SC202, a novel class I HDAC inhibitor, against colorectal cancer cells. Tumour Biol. 2016;37:10257-67.

14. Messerli SM, Hoffman MM, Gnimpieba EZ, Kohlhof H, Bhardwaj RD. 4SC-202 as a Potential Treatment for the Pediatric Brain Tumor Medulloblastoma. Brain Sci. 2017;7.

15. Mishra VK, Wegwitz F, Kosinsky RL, Sen M, Baumgartner R, Wulff T, Siveke JT, Schildhaus HU, Najafova Z, Kari V, Kohlhof H, Hessmann E, Johnsen SA. Histone deacetylase class-I inhibition promotes epithelial gene expression in pancreatic cancer cells in a BRD4- and MYC-dependent manner. Nucleic Acids Res. 2017;45:6334-49.

16. Inui K, Zhao Z, Yuan J, Jayaprakash S, Le LTM, Drakulic S, Sander B, Golas MM. Stepwise assembly of functional C-terminal REST/NRSF transcriptional repressor complexes as a drug target. Protein Sci. 2017;26: 997-1011.

17. Gruber W, Peer E, Elmer DP, Sternberg C, Tesanovic S, Del Burgo P, Coni S, Canettieri G, Neureiter D, Bartz R, Kohlhof H, Vitt D, Aberger F. Targeting class I histone deacetylases by the novel small molecule inhibitor 4SC-202 blocks oncogenic hedgehog-GLI signaling and overcomes smoothened inhibitor resistance. Int J Cancer. 2018;142:968-75.

18. Fu M, Wan F, Li Z, Zhang F. 4SC-202 activates ASK1-dependent mitochondrial apoptosis pathway to inhibit hepatocellular carcinoma cells. Biochem Biophys Res Commun. 2016;471:267-73.

19. von Tresckow B, Sayehli C, Aulitzky WE, Goebeler ME, Schwab M, Braz E, Krauss B, Krauss R, Hermann F, Bartz R, Engert A. Phase I study of domatinostat (4SC-202), a class I histone deacetylase inhibitor in patients with advanced hematological malignancies. Eur J Haematol. 2019;102:163-73.

20. Schuchmann M, Schulze-Bergkamen H, Fleischer B, Schattenberg JM, Siebler J, Weinmann A, Teufel A, Worns M, Fischer T, Strand S, Lohse AW, Galle PR. Histone deacetylase inhibition by valproic acid down-regulates c-FLIP/CASH and sensitizes hepatoma cells towards CD95- and TRAIL receptormediated apoptosis and chemotherapy. Oncol Rep. 2006;15:227-30.

21. Livak KJ, Schmittgen TD. Analysis of relative gene expression data using real-time quantitative PCR and the 2(-Delta Delta C(T)) Method. Methods. 2001;25:402-8.

22. Liao $B$, Zhou $H$, Liang $H$, Li C. Regulation of ERK and AKT pathways by hepatitis $B$ virus $X$ protein via the Notch1 pathway in hepatocellular carcinoma. Int J Oncol. 2017;51:1449-59.

23. Chen Y, Kamili A, Hardy JR, Groblewski GE, Khanna KK, Byrne JA. Tumor protein D52 represents a negative regulator of ATM protein levels. Cell Cycle. 2013;12:3083-97.

24. Shrivastav M, De Haro LP, Nickoloff JA. Regulation of DNA double-strand break repair pathway choice. Cell Res. 2008;18:134-47.

25. Regel I, MerkI L, Friedrich T, Burgermeister E, Zimmermann W, Einwachter H, et al. Pan-histone deacetylase inhibitor panobinostat sensitizes gastric cancer cells to anthracyclines via induction of CITED2. Gastroenterology. 2012;143:99-109 e110. 
26. Marchion DC, Bicaku E, Daud Al, Sullivan DM, Munster PN. In vivo synergy between topoisomerase II and histone deacetylase inhibitors: predictive correlates. Mol Cancer Ther. 2005;4:1993-2000.

27. Marchion DC, Bicaku E, Daud Al, Sullivan DM, Munster PN. Valproic acid alters chromatin structure by regulation of chromatin modulation proteins. Cancer Res. 2005;65:3815-22.

28. Thurn KT, Thomas S, Raha P, Qureshi I, Munster PN. Histone deacetylase regulation of ATM-mediated DNA damage signaling. Mol Cancer Ther. 2013;12:2078-87.

29. Munster P, Marchion D, Bicaku E, Schmitt M, Lee JH, DeConti R, et al. Phase I trial of histone deacetylase inhibition by valproic acid followed by the topoisomerase II inhibitor epirubicin in advanced solid tumors: a clinical and translational study. J Clin Oncol. 2007;25:1979-85.

30. Goder A, Emmerich C, Nikolova T, Kiweler N, Schreiber M, Kuhl T, et al. HDAC1 and HDAC2 integrate checkpoint kinase phosphorylation and cell fate through the phosphatase-2A subunit PR130. Nat Commun. 2018;9:764.

31. Yoon JY, Ishdorj G, Graham BA, Johnston JB, Gibson SB. Valproic acid enhances fludarabine-induced apoptosis mediated by ROS and involving decreased AKT and ATM activation in B-cell-lymphoid neoplastic cells. Apoptosis. 2014;19:191-200.

\section{Figures}


a

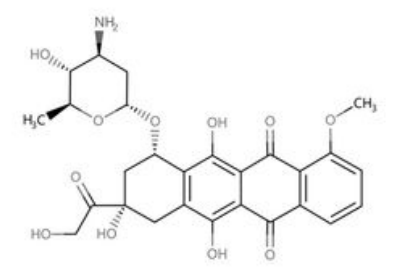

b

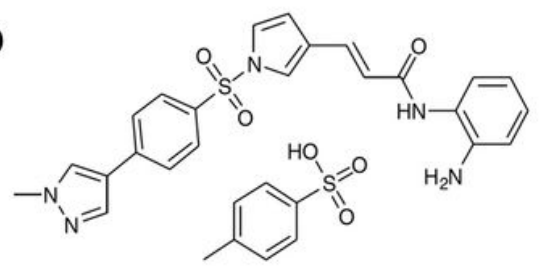

C

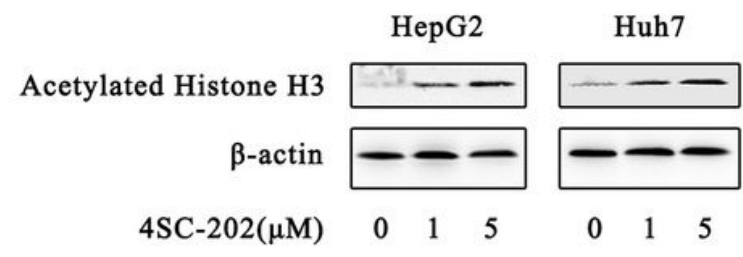

d

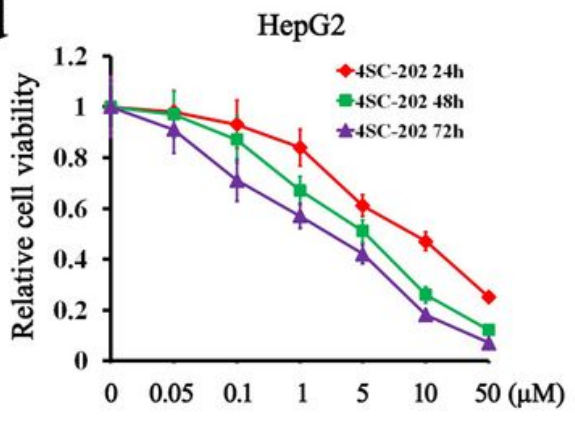

e

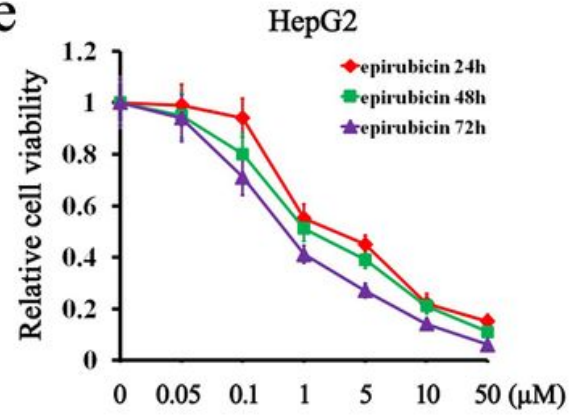

Huh7

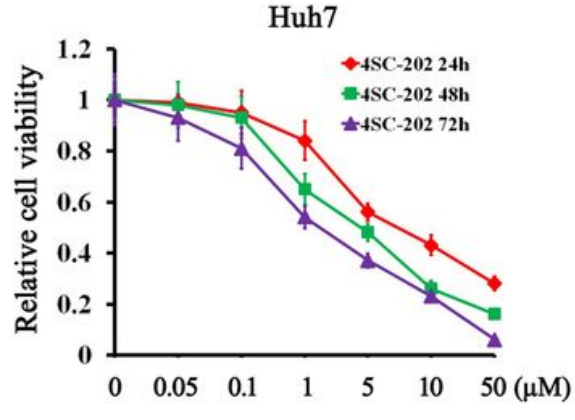

Huh7

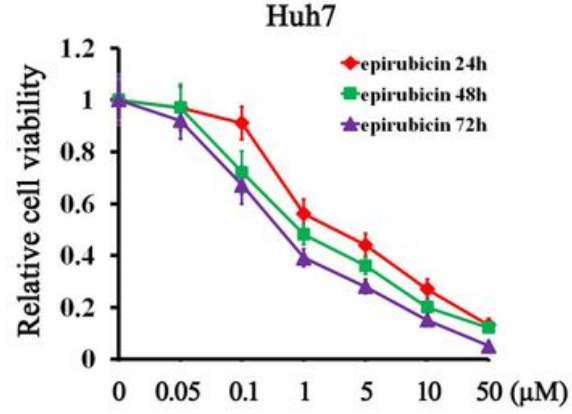

g

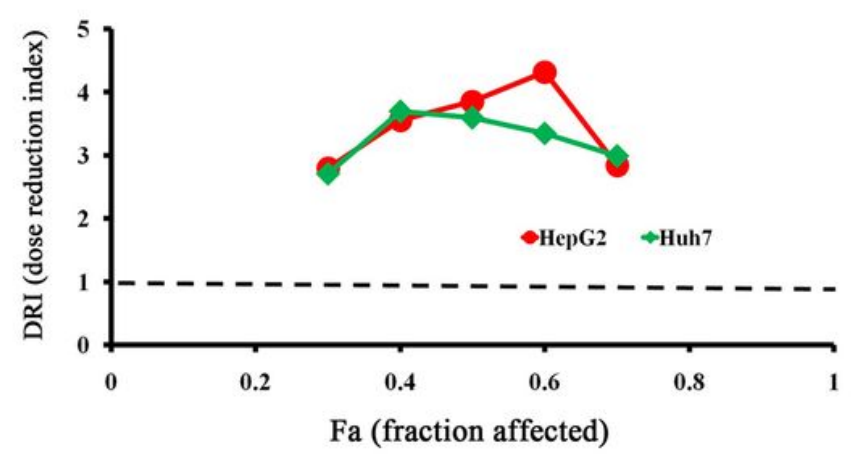

Figure 1

4SC-202 and epirubicin synergistically inhibit the proliferation of liver cancer cells. a Chemical structure of epirubicin. b Chemical structure of 4SC-202. c HepG2 and Huh7 cells were treated with different concentrations of 4SC-202 for $48 \mathrm{~h}$, and acetylated histone H3 was detected by western blotting. d, e HepG2 and Huh7 cells were treated with indicated concentrations of 4SC-202 or epirubicin for various times. Cell viability was tested by CCK-8 assay. f Combination index values of HepG2 and Huh7 cells at different fractions affected. g Dose-reduction index values of HepG2 and Huh7 cells at different fractions affected. 
a
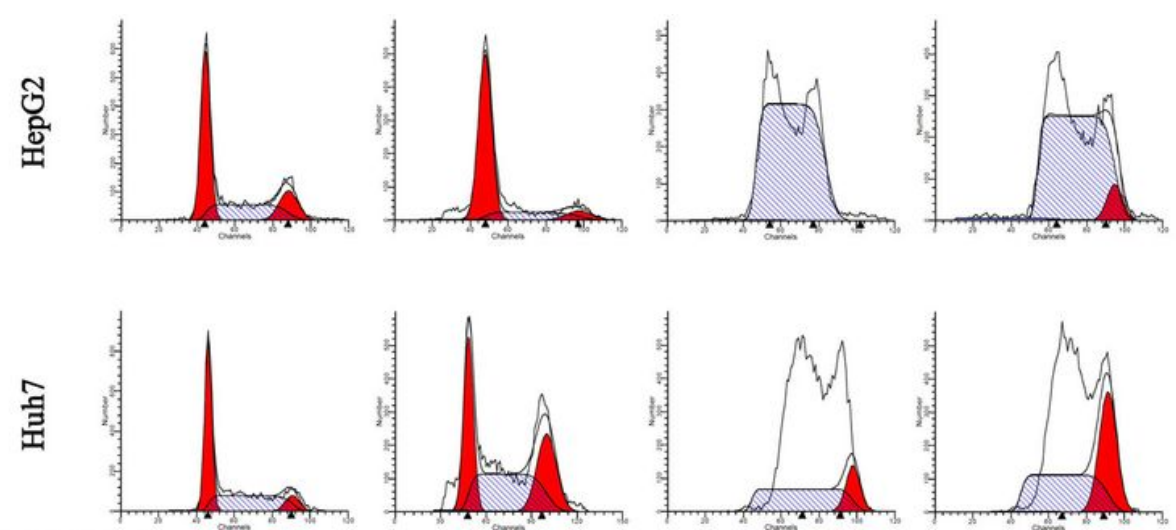

4SC-202 $(5 \mu \mathrm{M})$ epirubicin $(1 \mu \mathrm{M})$

b
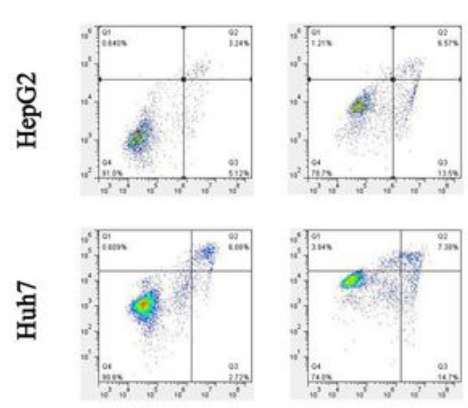

4SC-202 (5 $\mu \mathrm{M})$

epirubicin $(1 \mu \mathrm{M})$

d
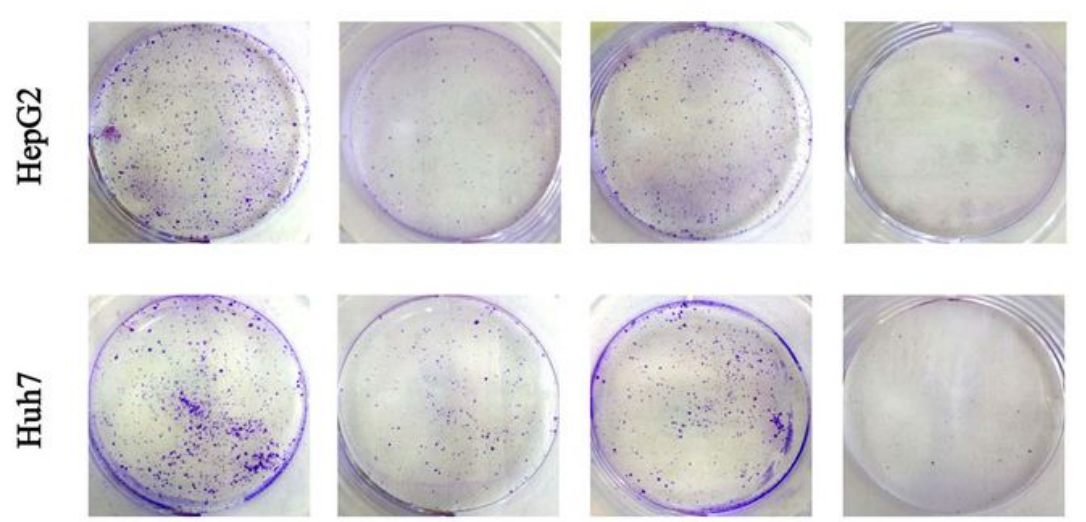

4SC-202 $(5 \mu \mathrm{M})$

epirubicin $(1 \mu \mathrm{M})$

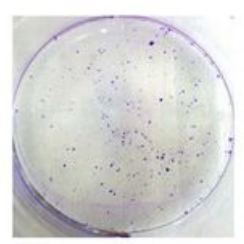

$+$

-

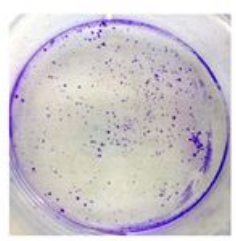

$+$
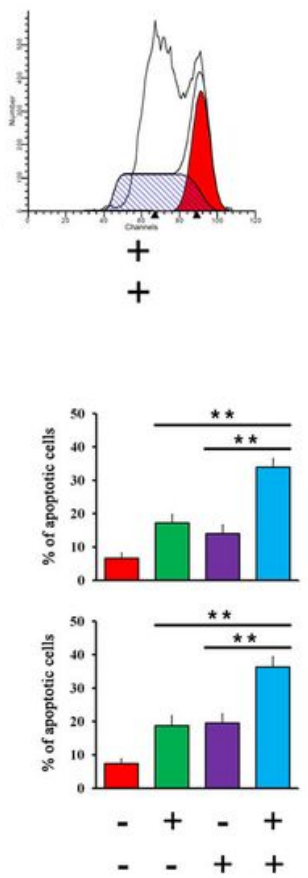
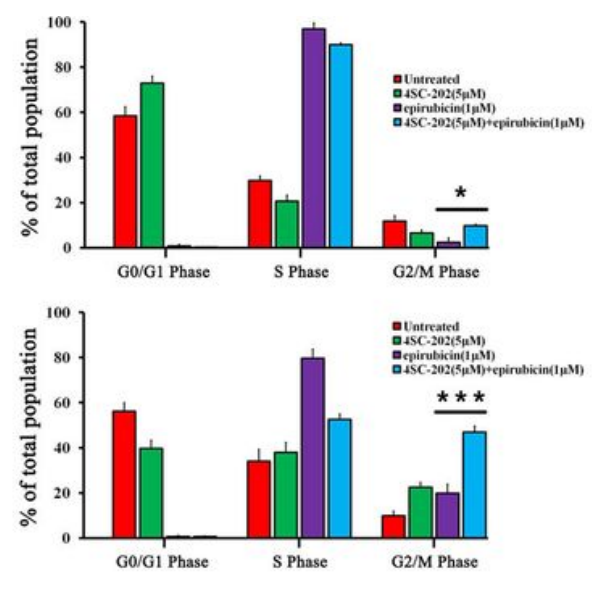

C
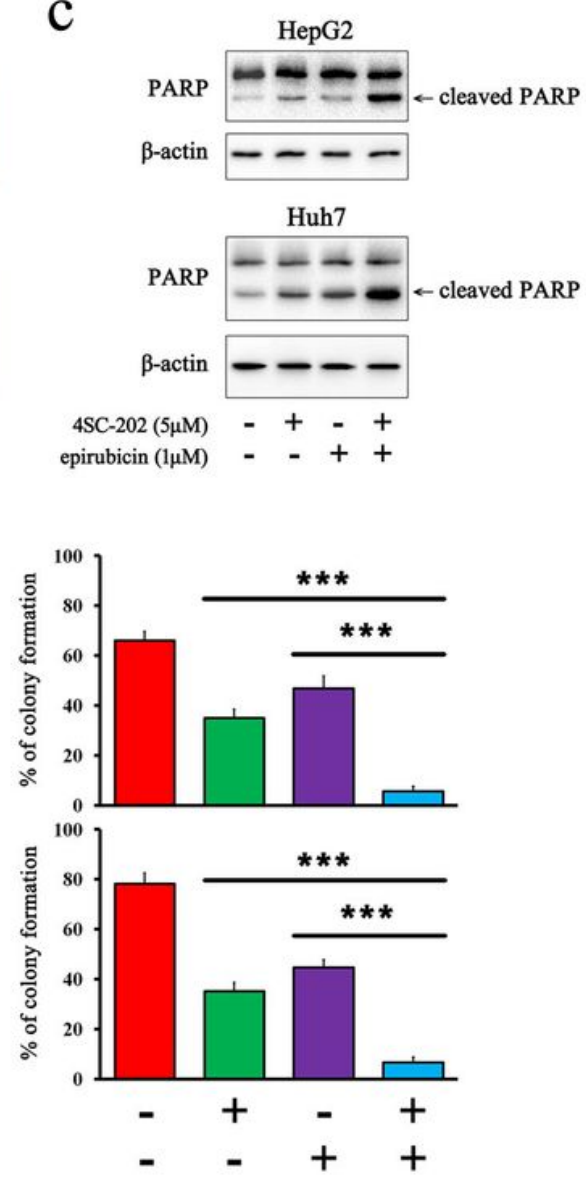

\section{Figure 2}

4SC-202 and epirubicin synergistically cause cell cycle arrest, elevated apoptosis, and reduced tumorigenicity in vitro. a HepG2 and Huh7 cells were treated with 4SC-202 $(5 \mu \mathrm{M})$ and epirubicin $(1 \mu \mathrm{M})$ for $48 \mathrm{~h}$ and cell cycle analysis was performed. b HepG2 and Huh7 cells were treated with 4SC-202 (5 $\mu \mathrm{M})$ and epirubicin $(1 \mu \mathrm{M})$ for $48 \mathrm{~h}$ and the apoptotic rate was determined. c Western blot analysis of cleaved-PARP in HepG2 and Huh7 cells treated as indicated for 48 h. d HepG2 (500 cells/well) and Huh7 (500 cells/well) cells were seeded in 6-well plates and treated with 4SC-202 $(5 \mu \mathrm{M})$ and epirubicin $(1 \mu \mathrm{M})$ 
for $48 \mathrm{~h}$. All the wells were stained and images were acquired on day 14 . Colony formation rate was calculated. ${ }^{*} \mathrm{P}<0.05, * * \mathrm{P}<0.01,{ }^{* * *} \mathrm{P}<0.001$.
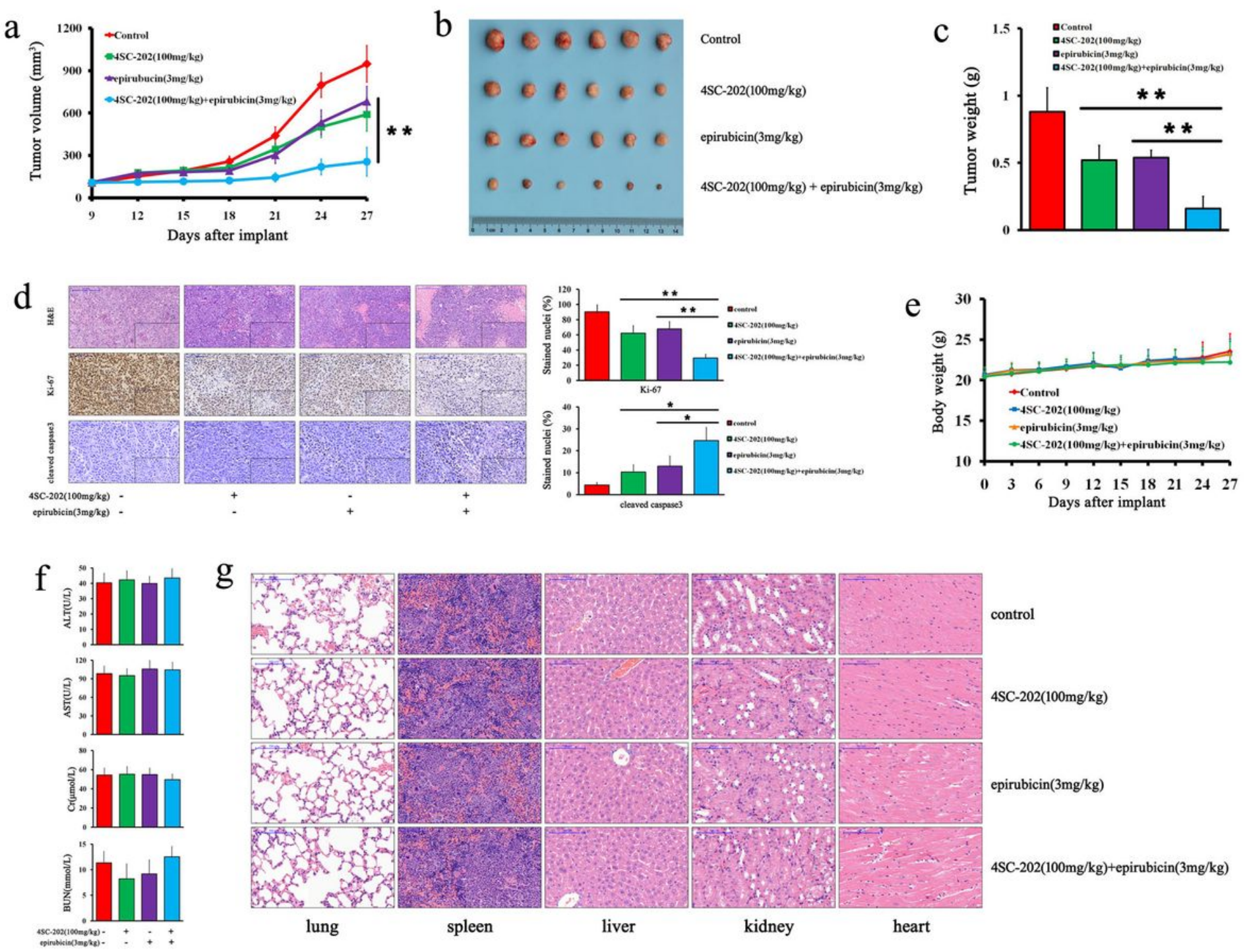

$4 \mathrm{SC}-202(100 \mathrm{mg} / \mathrm{kg})$

epirubicin(3mg/kg)

$4 \mathrm{SC}-202(100 \mathrm{mg} / \mathrm{kg})+$ epirubicin $(3 \mathrm{mg} / \mathrm{kg})$

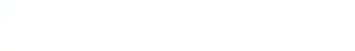

\section{Figure 3}

4SC-202 and epirubicin synergistically decrease tumorigenicity in vivo. On day 9 after subcutaneous injection of HepG2 cells, treatment with 4SC-202 and epirubicin started and the tumor size was calculated every third day. 4SC-202 (100mg/kg) was dosed p.o. daily and epirubicin $(3 \mathrm{mg} / \mathrm{kg})$ was injected i.p. every third day. a Tumor growth curve of the animals. b Tumors from all groups on day 27. c Tumor weights of

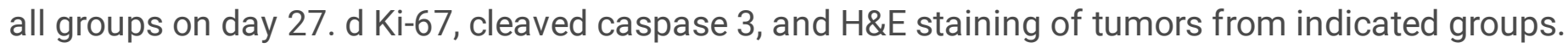
Quantitation of staining is shown on right. e Body weights of all groups. $f$ Biochemical functions of liver and kidney including AST, ALT, Cr, and BUN in serum were measured. g Representative images of H\&E staining of lung, spleen, liver, kidney, and heart in different groups. ${ }^{*} P<0.05,{ }^{*} \mathrm{P}<0.01$. 
a

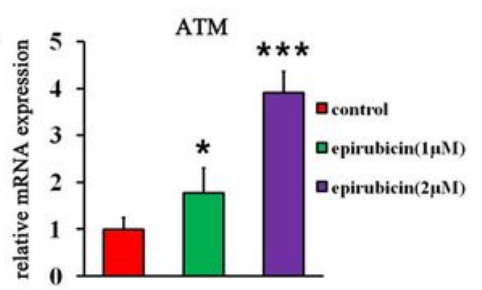

e

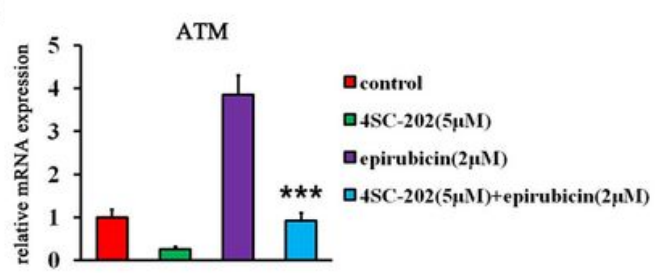

b

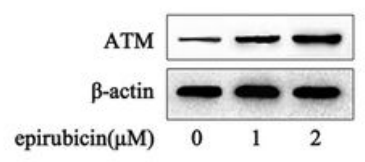

c

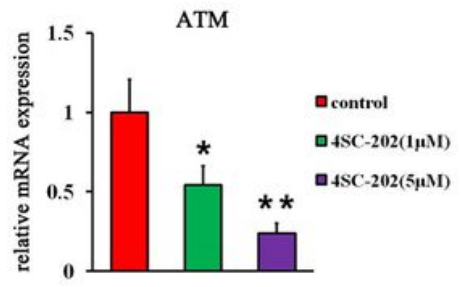

d

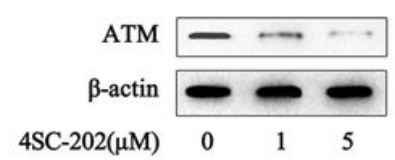

h
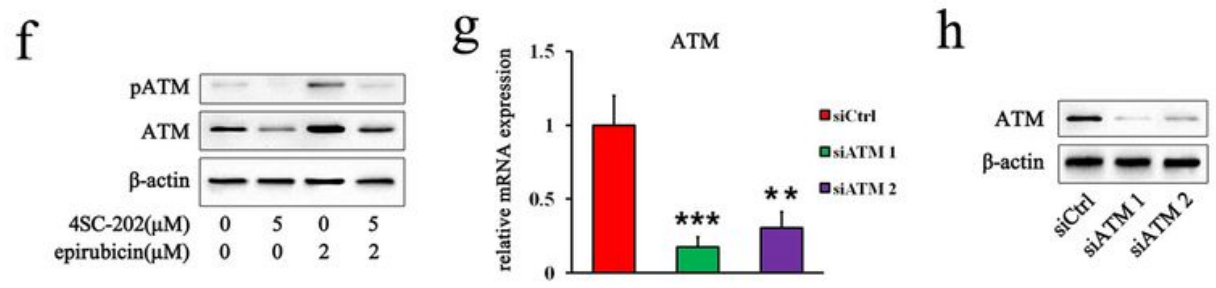

i
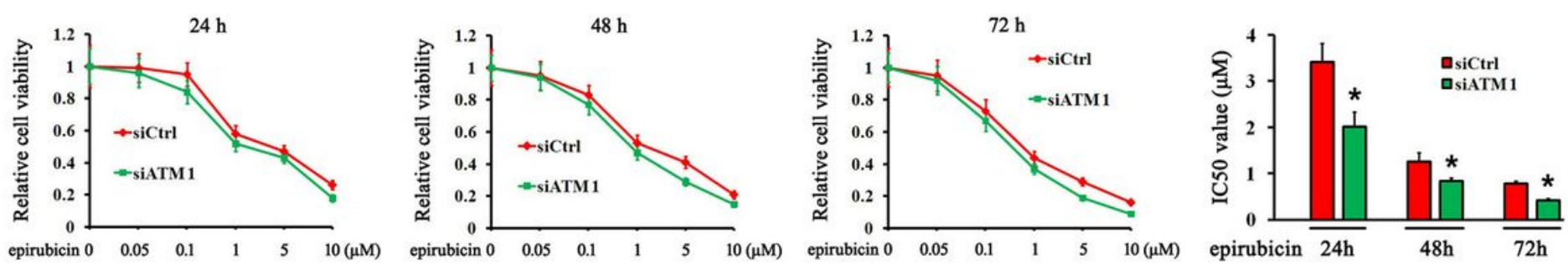

Figure 4

4SC-202 downregulates ATM expression induced by epirubicin in liver cancer cells. a qPCR of ATM mRNA in HepG2 cells treated with epirubicin. b Western blot analysis of ATM in HepG2 cells treated with epirubicin. c qPCR of ATM mRNA in HepG2 cells treated with 4SC-202. d Western blot analysis of ATM in HepG2 cells treated with 4SC-202. e qPCR of ATM mRNA in HepG2 cells treated with 4SC-202 and epirubicin. $f$ Western blot analysis of ATM and pATM in HepG2 cells treated with 4SC-202 and epirubicin. $\mathrm{g}, \mathrm{h}$ Inhibition of ATM expression by siRNA was confirmed by qPCR and western blotting. i Relative viability of HepG2 cells after treatment with epirubicin and ATM siRNA. IC50 values were analyzed. *P < $0.05, * \star P<0.01, * \star * P<0.001$. 

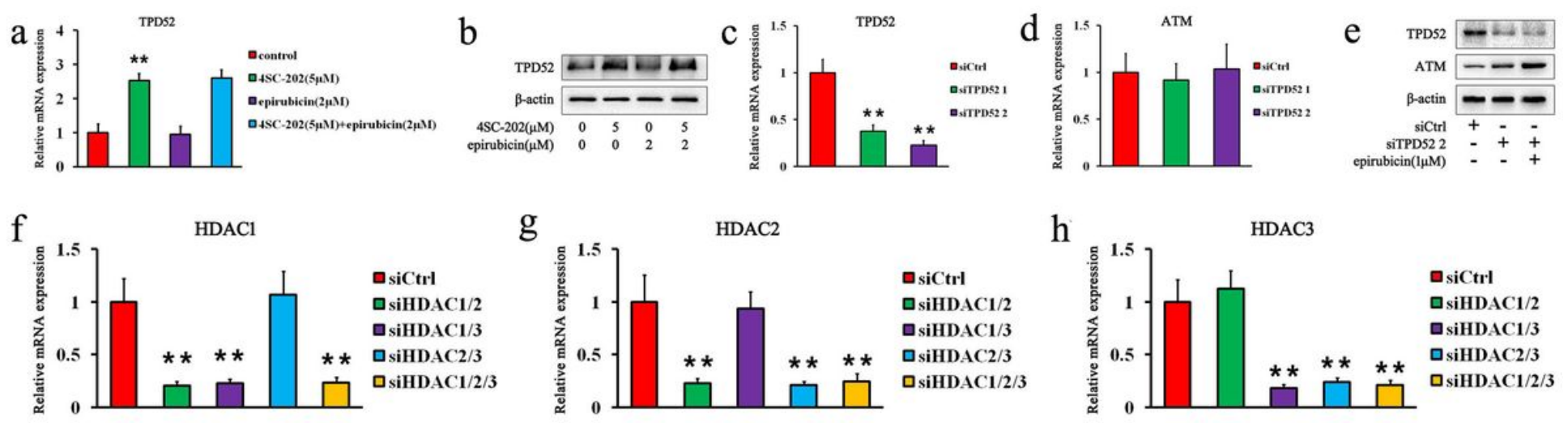

口iCtrl

口siHDAC1/2

口iHDAC1/3

口 $\mathrm{siHDAC2} / 3$

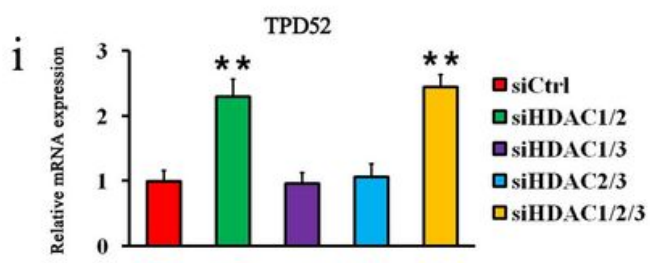

$\mathrm{k}$

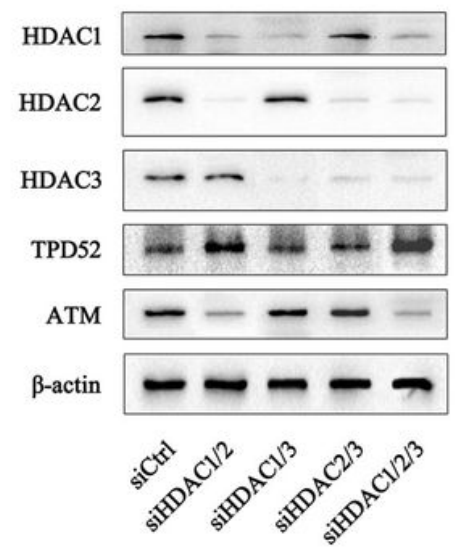

\section{Figure 5}

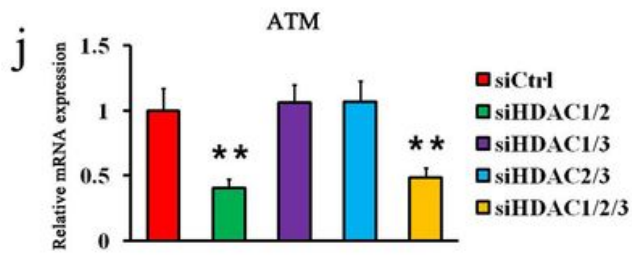

口siHDAC1/2/3

TPD52 is repressed by HDAC1/2 and downregulates ATM. a qPCR of TPD52 mRNA in HepG2 cells treated with 4SC-202 and/or epirubicin. b Western blot of TPD52 in HepG2 cells treated with 4SC-202 and/or epirubicin. c Inhibition of TPD52 expression by siRNA was confirmed by qPCR. d qPCR of ATM mRNA after TPD52 siRNA. e Western blot of TPD52 and ATM in HepG2 cells transfected with TPD52 siRNA. f-h qPCR of HDAC1, HDAC2, and HDAC3 mRNA in HepG2 cells transfected by indicated siRNAs. i qPCR of TPD52 mRNA in HepG2 cells transfected with HDAC1, HDAC2, and HDAC3 siRNA as indicated. $j$ qPCR of ATM mRNA in HepG2 cells transfected with HDAC1, HDAC2, or HDAC3 siRNA. $k$ Western blot of HDAC1/2/3, TPD52, and ATM in HepG2 cells transfected with HDAC1/2/3 siRNA. **P $<0.01$. 
$\longrightarrow$ Sequences for 5'primer's binding

GTCCCCACCAGATTCAGGAGCCCACCTGGCTTCACCTAGTGGATCCCGCACCGGGCCTGCAGGTGGAGCTGC CTGCCAGTCCCGCGCCATGCGCTCGCATTCCTCAGCCCTTGGGTGATCGATGGGACTGGGCGCCGTGGAGCA

Region A

GGAACCCACTAGCTACCCTG

GGGGGCAGGCATTCGTCGGGGAGGCTCGGGCTGCACAGGAACCCACGGAGGCGGGGGAAGGCTCAGGCAT GGCGGGCTGCGGTCCCGAGGCCTGCCCCGTGGGAAGGCAGCTAAGGCCCGGCGAGAAATCGAGCGCAGCG CCGGTGGGCTGGCACTGCTGGGGGACGCAGTACACCCTCCGCAGCCGCTGGCCCGGGTGCTAAGCCCCTCA CTGCCCGGGGCCGGCAGAGCCGGCCGGCCGCTCCGAGCGCGGGGCCCGCCAAGCCCACGCCCACCCGGAA CTCCAGCTGGCCCGCAAGCGCTGCTCGCAGCCCCGGTTCCTGCTCGCGCCTCTCCCTCCACACCTCCCTGCA AGCTGAGGGAGCCGGCTCTGGCCTTGGCCAGCCCAGAAAGGGGCTCCCACAGTGCAGCGGCGGGCCGAAG GGCTCCTCAAGTGCCGCCAAAGTGGGAACCCAGGCAGAGGAAGCGCCGAGAGCAAGAGAGGGCTCTGAGG $\longrightarrow$ Sequences for 5' primer's binding ACTGCCAGCACACTGTCACCTCTCACTCCCACTTCGGCCTCCCAAAGTGCTTGGATGACTGGCATGAGCCAC Sequences for 3' primer's binding TGTGCCCGGCTCAGAATTTCTTTTTGGGGTGATGAAAATGCTCAAAGTTAGATTACGGTGATGGTTGCGCGCC

Region B CTAATGCCACTACCAACGCG TCTGTGAATATACTCAAAAGCCTTGAAAACAAAAGTTGAGGTGCTACAAACTAAAGGATCTAATCGCAGTCAC

$\rightarrow$ Sequences for 5 ' primer's binding AGAACAGTTGCCCATGCTTAGTGGCAAGAGGCTGTATCCTGAGGACTTTTGTGTTTCCAGATTTCTCCAAAGG Sequences for 3' primer's binding 7
AGAATTACAGTTTGGCAGGTGGAATGAGGGTCAGGTAGAAAAGGCTGACGATGCAACTAACTTAAGGAGGG Region C TCCGACTGCTACGTTGATTG GGAGGACAAAGGGTGAAGACCAGGTCAGTGTGGAGAAAGGGAGGTTGTAGGATGTT

: 3' primer used for ChIP in the TPD52 promoter

$\mathrm{c}$

b

TPD52

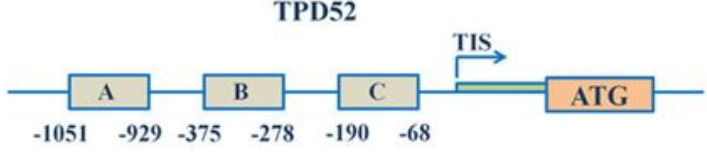

d

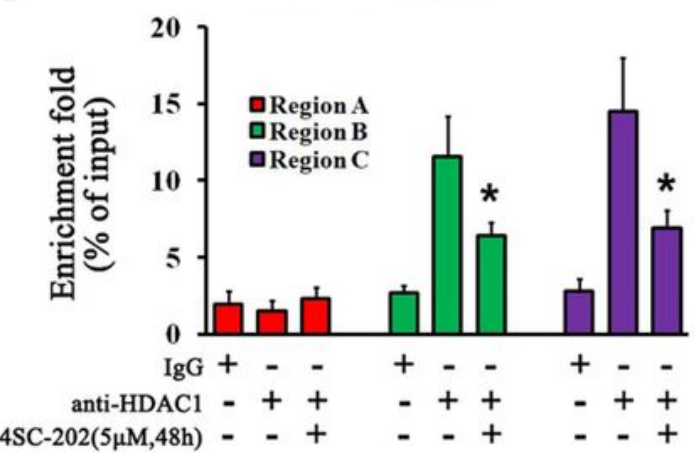

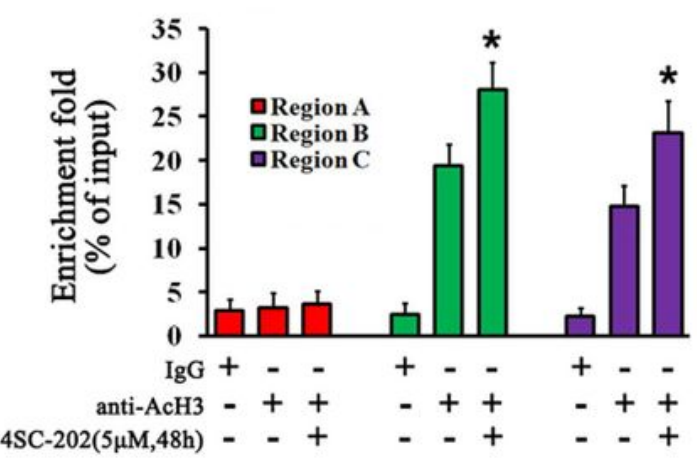

e

TPD52

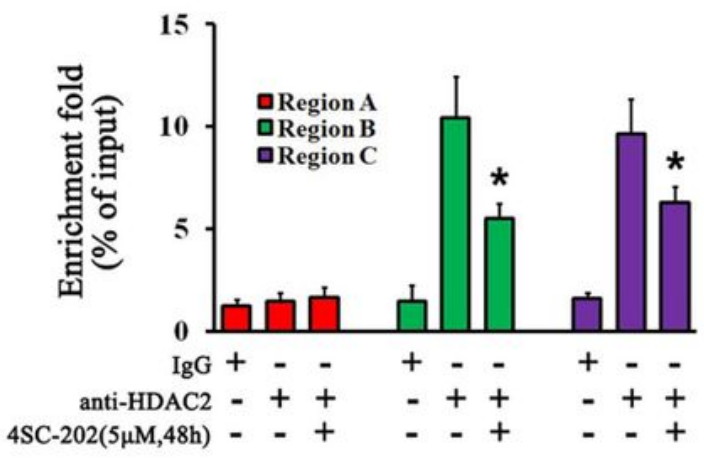

\section{Figure 6}

AcH3 and HDAC1/2 competitively bind to the TPD52 promoter. a TPD52 promoter. Sequences of primers are in blue letters. $b$ Schematic diagram of the TPD52 promoter region. $c$ ChIP assay was performed to evaluate the binding of $A c H 3$ to the promoter of TPD52. Three different DNA regions $(A, B$, and $C)$ from the TPD52 promoter were analyzed by qPCR. Cells were treated with 4SC-202 or vehicle. d, e ChIP assay 
was performed to evaluate the binding of HDAC1 and HDAC2 to the promoter of TPD52. ${ }^{*} \mathrm{P}<0.05,{ }^{*} \mathrm{P}<$ 0.01 .

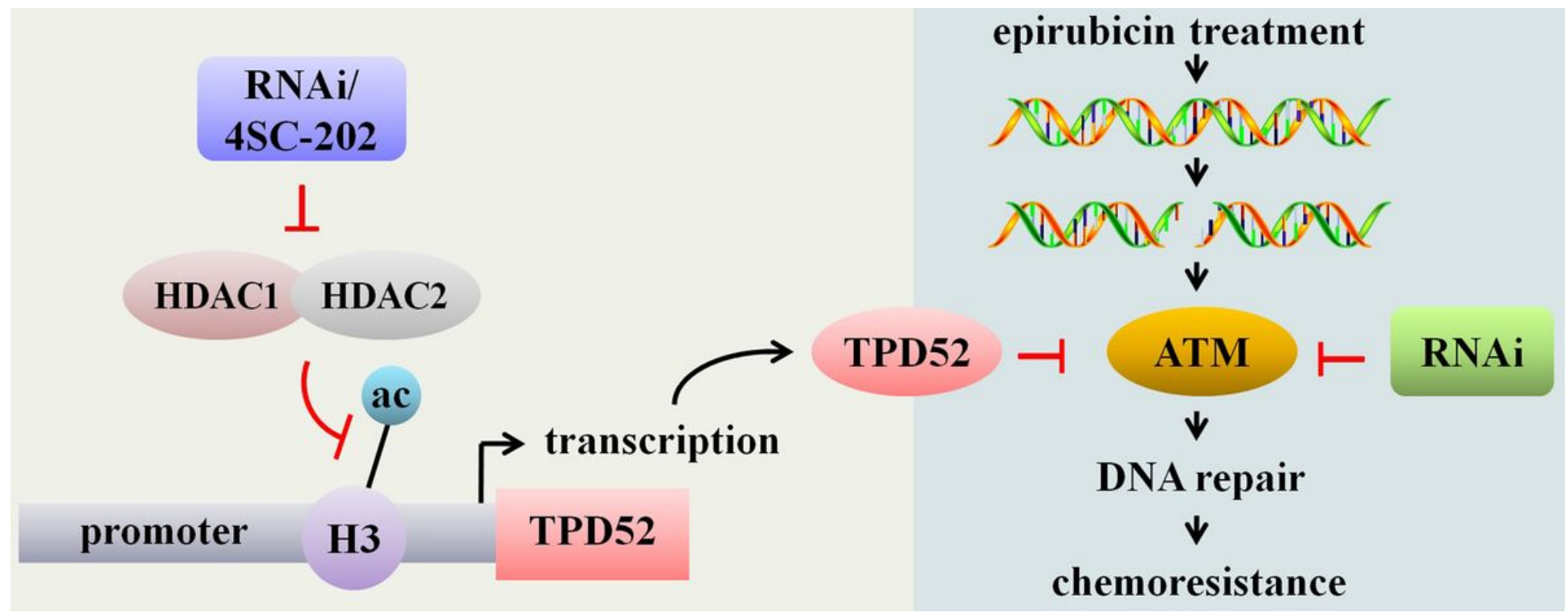

Figure 7

Overview of the molecular mechanism by which 4SC-202 increases sensitivity to epirubicin in liver cancer cells. 